\title{
La cuestión de la identidad Nath: Consideraciones sobre una orden de yoguis
}

\author{
Adrián Muñoz ${ }^{1}$ \\ El Colegio de México (México)
}

Recibido: septiembre 21 de 2015. Revisado: noviembre 20 de 2015. Aceptado: diciembre 12 de 2015

Referencia formato APA: Muńoz, A. (2016). La cuestión de la identidad Nath: Consideraciones sobre una orden de yoguis. Rev. Guillermo de Ockham, 14(1), 79-87. doi: http://dx.doi.org/10.21500/22563202.2307

\section{Resumen}

Es una constante en la historia religiosa del sur de Asia la presencia de distintos grupos de renunciantes y yoguis, entre los cuales una pieza crucial ha sido el grupo de ascetas comúnmente conocido como Nāth Panth o Nāth Yoguis. Su relevancia deriva tanto de su participación en la historia premoderna y moderna de la política en el sur de Asia, como de sus contribuciones para la formación del panorama cultural y religioso de las sociedades surasiáticas. El presente artículo se propone revisar algunos aspectos y problemáticas relativas a la identidad nāth. Así, cuestionará lo que significa ser un yogui -un nāth-y se referirá a fuentes originales para examinar lo que dicen al respecto considerando para ello las herramientas textuales y aproximaciones posibles para hacerlo.

Palabras clave: Nāths, yoguis, identidad, yoga, India, religión y sociedad

\section{The question of the Nath's identity: Considerations about an order of Yogis}

\begin{abstract}
Diverse groups of yogis have played significant roles in the religious history of South Asia. A special piece in this wide landscape is the group of ascetics that is traditionally known as the Nāth Panth, or the order of the Nāth Yogis. Their importance lies not only in the political history of both pre-modern and modern South Asia, but also in the making up of the cultural and religious outline of various South Asian societies. This paper seeks to revisit some aspects and problems concerning the so-called Nāth identity. It will discuss what it means to be a yogi, a Nāth, and what do original sources have to say on this, as well as consider the textual materials and possible approaches to achieve this purpose.
\end{abstract}

Keywords: Näths, yogis, identity, yoga, India, religion and society

1. Especialista en tradiciones religiosas del sur de Asia, Adrián Muñoz ha impartido clases sobre religiones y tradiciones literarias comparadas, temas sobre los que ha publicado numerosos artículos especializados. Es autor de La piel de tigre y la serpiente: La identidad de los nath-yoguis a través de sus leyendas (2010) y Los versos satánicos de Blake (2012). Y su reciente libro, Radiografía del hathayoga, publicado poe el Centro de Estudios de Asia y África El Colegio de México C.P. 10740. E-mail: amunoz@colmex.mx 


\section{A questão da identidade Nath: Considerações sobre uma orden de yoguis}

\section{Resumo}

A história religiosa do sul da Asia há contado com a presença de diferentes grupos de ascetas comunmente conhecido como Nāth Panth o Nāth Yoguis. Sua relevância deriva de sua participação na história pré-moderna e moderna da política no Sul da Ásia, como de suas contribuiçóes para a formação de paisagem cultural e religiosa das sociedades do sul da Ásia. O presente ensaio propone-se revisar algums aspectos e problematicas relativas á identidade Nath. Assim, vai questionar o que significa ser um Yogui, um Nath, e se referem a fontes originais para examinar qué dicen sobre o assunto, além de considerar as ferramentas textuais e abordagens possíveis para realizá-lo.

Palavras-chave: Nāths, yoguis, identidade, yoga, India, religión e sociedade

\section{Introducción}

Las figuras del asceta y el renunciante son componentes ineludibles del mosaico cultural y social de la India. Entre estos, los yoguis poseen un lugar destacado que, sin embargo, no ha estado exento de ambigüedades y tensiones; incluso la manera de distinguirlos de otros renunciantes puede ser complicada. Existen diversos tipos de yoguis no solo porque también existen distintos tipos de yoga, sino también por los distintos papeles que los yoguis desempeñan en sus contextos particulares. Los nāths o nāth-yoguis, conforman un grupo de particular interés gracias a que han logrado permanecer en el imaginario colectivo durante años. Por otro lado, algunas ramas de la orden también han conseguido ejercer una influencia considerable en los devenires políticos de la India moderna y contemporánea.

Interesa discutir en este escrito un par de situaciones y problemáticas que giran en torno a la construcción de la identidad nāth. Los nāths constituyen una orden religiosa de yoguis nominalmente devotos del dios Śiva, usualmente asociados a la práctica del hațhayoga y cuya presencia es más fuerte en el norte del subcontinente asiático. De manera general, podemos situar el surgimiento de la orden hacia los siglos XII-XIII y las referencias a ellos son altamente frecuentes en todo tipo de literatura y en distintas lenguas.

Ahora bien, para referirse concretamente a una "construcción de la identidad" se debe entender que la identidad bien puede ser un compuesto de proyecciones propias (ideología, teleología, etc.), como de aportaciones ajenas (percepción, aceptación o rechazo). La identidad nāth ciertamente está compuesta de esta última manera, pues en gran medida se ha conformado por elementos que surgen de textos escritos y orales, de ideales religiosos y de realidades sociales.

Sobre este punto, interesa destacar una discusión reciente en los estudios sobre los nāths. Mallinson (2011) y otros son de la opinión de que los apelativos nāth y yogui se convirtieronen términos sinonímicos hacia el siglo XVIII, en parte gracias a los británicos. El autor sugiere, incluso, que la etiqueta nāth-yogui no se emplea antes del siglo XVIII para referirse a una orden religiosa bien definida y señala que la palabra nāth (en sánscrito o hindi) se refería siempre a la divinidad suprema (usualmente Śiva) (Mallinson, 2012). Las referencias más tempranas a una orden nāth -señala- datan del siglo Xviı. Estudiosos como White (2009) comparten esta postura. El asunto es no es solo interesante sino también importante para entender las relaciones entre sociedad y movimientos religiosos durante los siglos XII y XVIII. En buena medida, la ambigua cuestión de la identidad nāth depende, como lo señala Mallinson, de la carencia de estudios centrados en la historia de estos yoguis. Hay especialistas que han llevado a cabo estudios etnográficos, traducido manuales en sánscrito o trabajado material folclórico, pero muy pocos, hasta la fecha, se han dedicado a la parte más historiográfica.

\section{Aproximaciones posibles}

Para esta y otras temáticas se sugiere una aproximación doble al problema. La cuestión de la identidad nāth se puede abordar mediante una pesquisa historiográfica o a través de una indagación hermenéutica. Comúnmente se trata de perspectivas excluyentes, pero se podría obtener un panorama más completo si combinamos dichos esfuerzos. No es la intención aquí llevar a cabo una defensa teórica de estas aproximaciones; antes bien, lo que interesa es poner sobre la mesa el hecho de que un solo acercamiento puede 
hacer perder algún detalle importante. Importa, además, puntualizar cuáles son los obstáculos inherentes y a qué tipo de material se puede recurrir.

La pesquisa historiográfica, desde luego, será la que procure rastrear evidencias sólidas. La dificultad más importante para ello radica en el hecho de que las fuentes para ese tipo de escrutinio son o bien escasas o difíciles de manejar, pues requieren el conocimiento lingüístico de al menos tres lenguas y el auxilio de la arqueología y la epigrafía. Evidentemente, el estudioso no siempre podrá contar con dicho conocimiento y en consecuencia, tendrá que depender en distintos grados de fuentes secundarias o derivar información a través de terceros, a saber: arqueólogos y epigrafistas. Afortunadamente, hay una literatura considerable (nunca suficiente, sin embargo) que ha proporcionado datos relevantes para comprender los papeles, las participaciones y las actuaciones de los yoguis en diferentes ámbitos y momentos históricos.

De manera preponderante, resultaría provechoso acudir a los registros de los protagonistas políticos y culturales de los siglos XII y XVIII, razón por la cual las fuentes de las cortes mogolas y otros gobiernos de la península podrían ser de utilidad. Se sabe que algunos dirigentes mogoles tuvieron acercamiento a yoguis y otros ascetas, aspecto que conllevaría averiguar más a fondo la naturaleza de estos encuentros y las maneras específicas como los unos se dirigían a los otros. Estos documentos pueden arrojar luz sobre los sitios que revisten particular importancia para las órdenes de yoguis y los apelativos que estos utilizaban.

La indagación hermenéutica puede ser más de tipo comparativo e interdisciplinario. No solo se basa en determinados tipos de documentos, sino que recurre a distintas narrativas y a sus variantes. Puede también referirse a documentos históricos para dar un contexto amplio a la interpretación de un ámbito específico, pero concede una importancia particular a entornos simbólicos que se pueden materializar en artículos palpables y duraderos en la sociedad, como templos, instituciones y material cultural (por ejemplo, las estampas populares). La lectura de las leyendas y los mitos de una tradición religiosa revela, por ejemplo, factores ideológicos; la identificación del mitema apunta hacia una experiencia compartida. Además, la confrontación de variantes refleja o bien inconsistencias o bien sensibilidades regionales, pero también el posible intercambio con otras tradiciones religiosas.

Desde luego, hay un margen de error en el sentido de que las proyecciones simbólicas o ideológicas vertidas en una imagen pueden no derivar de manera directa de una circunstancia histórica comprobable, sino de inter- pretaciones simbólicas de la historia. Sin embargo, ello no implica que estas "proyecciones" carezcan de peso y relevancia. El hecho de que existan imágenes, cultos o estampas populares dice mucho acerca de las construcciones sociales de sensibilidades religiosas y de las maneras como estas conciben su vínculo con un pasado real o legendario (por ello no es necesario corroborar la historicidad de Jesús de Nazareth para estudiar aspectos varios del cristianismo, por mencionar un punto de analogía.) La confluencia de todo este tipo de material, finalmente, puede ayudarnos a dar perspectiva a continuidades o transformaciones identitarias. La combinación de material legendario o hagiográfico con registros históricos originales, proporciona, como apunta Pinch (2012), "[...] una idea de cómo los significados relacionados con acontecimientos, personas y sitios diversos del finales del siglo xvi se han filtrado en la comunidad de näth yoguis a lo largo del tiempo" (pp. 174-275).

\section{Los nombres}

El apelativo nāth-yogui-según señalan algunos especialistas- se concibe o define hacia el siglo XviII. Ciertamente, hay que tener en cuenta que la palabra nāth o nātha figura en textos anteriores a la llegada de los británicos, quienes no inventaron la palabra. Se trata de una voz sánscrita que significa "señor, amo, refugio, protector". Aparece frecuentemente como parte del nombre de personajes divinos, como es el caso de Jagannāth, la deidad tutelar de Orissa. Ha sido, además, un apelativo comúnmente ańadido al nombre iniciático de un adepto o devoto de alguna de las distintas ramas religiosas en el sur de Asia.

El nāth-yogui es un tipo de renunciante que se diferencia de otros grupos ascéticos en buena medida gracias a los kundal, los peculiares aretes que portan en ambas orejas. Este distintivo no solo indica que el adepto es un renunciante, sino también que ha recibido la iniciación y ha pasado a formar parte de la orden de yoguis que se asumen seguidores de las enseńanzas de Gorakhnāth, de tal suerte que en el imaginario colectivo de la península, yogi es frecuentemente sinónimo de gorakhnäthi; es decir, seguidor de Gorakhnāth. Gorakh fue una figura semilegendaria que pudo haber vivido alrededor del siglo XII, pero no hay pruebas concluyentes al respecto.

El apelativo de yogì, o su forma vernácula jogī, suele referirse, entonces, a los gorakhnāthis sobre todo a partir de la India premoderna. Otro epíteto o nombre con frecuencia utilizado para designarlos es siddha. De este modo, al consultar diversas fuentes, tanto en el folclor como en la 
tradición textual nāth, yogī y siddha a menudo funcionan como sinónimos, si bien en sentido estricto cada vocablo puede designar a personas distintas.

La palabra siddha quiere decir "alguien perfecto o que ha alcanzado la perfección [espiritual, ascética]". Un siddha (de la raíz $\sqrt{s i d h}$, tener éxito, perfeccionarse) es un asceta que ha logrado distintas perfecciones o "éxitos", los más famosos de los cuales son los ocho que se pueden adquirir gracias a la práctica del yoga. Nätha, por su parte, alude a "seńor, amo, protector, refugio" y en el presente contexto designa, por un lado, al militante de algunas de las órdenes iniciadas o vinculadas con Gorakhnāth y, por el otro, a quien ha controlado los sentidos mediante las prácticas psicofísicas del hațhayoga. Procede de la raíz $\sqrt{n a \bar{t} t}$, "poseer señorío, poder", pero también "implorar, suplicar". Empleado en sentido absoluto, Nātha puede, asimismo, designar al dios Śiva, en particular en el compuesto Ādinātha (lit. Nātha primordial) que suele figurar en las listas de maestros ejemplares del linaje nātha. Śiva, pues, constituye el primer nātha; es decir, el mejor controlador de los sentidos por medio de poderes yóguicos. Una excepción interesante aparece en el Akulaviratantra, texto atribuido a Matsyendra y relativamente temprano (¿siglo IX e.c.?). Allí, en una clara referencia a Śiva -quien primero impartió los sagrados secretos-se utiliza la expresión siddha-nātha (Akulaviratantra, 2).

Así, los seguidores del Nāth Panth son conocidos como nāth-panthis, pero también como kānphațis (yoguis de "la oreja perforada”). Además de kānphațis también reciben el nombre de darśanīs, debido a uno de los nombres con que se conoce a los aretes (darśan) que portan muchos de los nāth-yoguis en la actualidad. Esta práctica posiblemente entró en vigor entre los siglos XvII-XviII. Vale la pena señalar que no todos ellos son necesariamente descendientes espirituales de Gorakh ni portan los aretes (estos son, estrictamente, los kānphața-yoguis, kānphatīs o gorakhnāthīs), pero la mayoría de yoguis, independientemente de su linaje en particular, se pueden referir a sí mismos como nāths o nāth-siddhas, apelativos genéricos favorecidos por ellos mismos en sus publicaciones. Con bastante frecuencia, los nāths son también conocidos y auto-concebidos como yoguis, siddhas o avadhūtas, términos que, en sentido genérico, se refieren a ascetas cuyas arduas prácticas espirituales y austeridades les han prodigado diversos poderes sobrehumanos (siddhis).

Como es de esperar, el término nātha también se explica en varias fuentes a partir de una etimología homilética. Así, el Rājaguhya declara que la sílaba nā connota la anādi, la forma primordial (lit. "sin origen"), mientras que la sílaba tha connota sthäpita, "establecido". Nätha, pues, significaría la forma o el dharma primigenio establecido en los tres mundos (bhuvanatrayam) (cf. Dvivedi, 1996, p.3). Esta explicación carece de fundamento gramatical, pero al menos deja entrever la importancia del término nātha, el cual desde luego merecía una dilucidación esotérica.

\section{Yoguis, historia y fuentes textuales}

La pesquisa historiográfica necesita escrutar en crónicas, registros y otros materiales textuales. Resulta importante indagar, por un lado, los momentos en los que la conformación o el prestigio de los nāth-yoguis se "consolidó"- por así decirlo-; y por el otro, rastrear las maneras específicas como las fuentes textuales históricas se referían a ellos. Parece probable que el culto de los nāths se practica en Bengala desde el siglo XI (Bhattacharya, 1996). El periodo de florecimiento parece ubicarse entre los siglos XIII y XVI, periodo en que se compusieron varios de los textos asociados a los nāths. Su supuesto declive habría comenzado en el siglo XviII (cf. Bouy, 1994; Dvivedi, 2004).

Un punto que parece significativo y sobre el cual es importante insistir, es el hecho de que los yoguis sostuvieron un intercambio intenso con diferentes grupos de sufíes, los cuales comenzaron a tener una presencia ponderable en la península desde el periodo del sultanato de Delhi. Esto resulta interesante no solo por la interacción entre comunidades musulmanas e hindúes, sino porque dichos encuentros, sobre todo en las regiones del norte de India, parecen haber estado cobijados por la cultura de la devoción o bhakti. Así, los sufíes indios hubieron de articular diferentes dinámicas de interlocución con sus pares indios, en particular con los sants (Muñoz, 2011). La transición entre el declive del sultanato de Delhi y el surgimiento del imperio mogol significó cambios en la configuración política del subcontinente, pero también hubo continuidades en la esfera cultural. Los sants, los yoguis y los sufíes habían llegado para quedarse.

Que los yoguis fueron un grupo importante entre los siglos XII y XVIII es un hecho comprobado por las innumerables referencias históricas y hagiográficas de la época. Por ejemplo, Madhava (siglo XIv) en su biografía de Śankara refiere una historia que involucra a dos de los personajes nāths más prominentes, a saber: Matsyendra y Gorakh. Por otra parte, los documentos sobre los yoguis de Jakhbar en el Punjab (a partir del siglo xvI), atestiguan que los mahants del establecimiento yogui recibieron allí varias donaciones de tierras y dinero a manos de diferentes gobernantes musulmanes, algunos de los cuales fueron 
Akbar, Jahangir, ShahJahan y Aurangzeb (Goswamy \& Grewal, 1967 passim). En una inscripción del templo de Naraini en Kalinjar, (ca. 1519, Uttar Pradesh) se leen los siguientes nombres: Prānanātha, Paṃganātha, Gopīnātha, Mānsiṃgh y Nandanasiṃgh (Prasad, 1990). Aunque la inscripción está deteriorada, parece que se trata de un registro de una donación o patronazgo (las dos personas llamadas Singh) a favor de unos yoguis, todos los cuales se llaman nāth. Quienes otorgan el favor no son musulmanes y no podemos saber su rango social, pero ciertamente es frecuente la relación gobernante-yogui.

Este tipo de fenómeno ha sido discutido por Gold (1992, 1995), pero aún queda mucho por explorar. Uno de los casos que Gold ha estudiado acerca de la relación entre yoguis y gobernantes se refiere a la influencia que un yogui llamado Ayas Dev Nath ejerció sobre Man Singh, rey de Mewar (Jodhpur), en Rajasthan, en el siglo XIx. Man Singh se convirtió en un gran patrón de los nāths al grado de conformar una gran e importante biblioteca que terminó por convertirse en los Archivos de Mehangarh dentro del fuerte de Jodhpur, ahora una de las colecciones más importantes de literatura nāth. En todo caso, Man Singh no era gobernante musulmán y corresponde a la historia más reciente. Para evaluar mejor las evidencias, es necesario remitirse al periodo del establecimiento del dominio islámico en el sur de Asia.

\section{Yoguis e islam}

En términos generales, la actitud que mantuvieron los regidores musulmanes, en su mayoría de orientación sunita, fue de rechazo a las prácticas religiosas autóctonas de la India, concretamente hindú y jaina. Si bien hubo momentos de relativa tolerancia durante el periodo del sultanato, el Estado oficialmente defendía la fe islámica por encima de las otras. $\mathrm{Al}$ mismo tiempo, el sultanato vehiculó la introducción del sufismo, un factor que parece haber sido importante para favorecer la conversión. Entre los siglos XII y XIV la interacción parece haber sido poca o reservada entre el islam y el hinduismo, pero una de las excepciones notables es la registrada entre sufíes y yoguis. Ibn Battuta (s. XIV), por mencionar una de las fuentes confiables, registró el interés que Muhammad Tugluq tuvo acerca del poder de los jogìs (Ernst, 2007).

Entre las órdenes sufí que llegaron a India, algunas tendían a ser más ortodoxas y celosas de la Shariah y otras se mostraron menos conservadoras y poco a poco se "indianizaron" en términos de expresión, como describen algunos especialistas (Sarkar, 1985). Parcialmente derivado de ello, los sufíes de la orden qalandariya llegaron a estar más expuestos a los yoguis (Gold, 1987), pero también la orden chishti. Gradualmente este ambiente habría de producir una suerte de periodo de síntesis en el cual algunos ismailíes, por ejemplo, incorporaron ciertos elementos vaișnavas y śaivas: desde la doctrina de los avatares, hasta algunas formas de yoga tántrico, lo cual se puede evidenciar en la literatura nizari (Khan, 2000). En el siglo Xvi, las dos órdenes que más influencia habían adquirido eran los chishtiyas y los suhrawardis, que a veces tenían que competir también con los qadiris y los shattaris (Aquil, 2007).

Un posible lugar común de encuentro parece haberse dado entre las clases bajas. Varios estudiosos han sugerido que las tradiciones nāths pudieron haber sido relevantes para la casta de tejedores, los juläha, que recién se había convertido al islam (Gold, 1987). De hecho, es posible que todavía algunos yoguis en Assam y Bengala estén asociados a este oficio (Bhattacharyya, 1996). En Uttar Pradesh, la secta de los BharthariJogis no solo pertenece a esta casta, sino que también son musulmanes de nacimiento (Champion, 1995). Frecuentemente se desenvuelven como bardos itinerantes, pero resulta significativo el hecho de que han moldeado las narrativas nāths, para adecuarse a una sensibilidad sufí.

En un valioso artículo, Ernst (2005) sugiere que posiblemente los yoguis fueron el único grupo religioso con el cual los sufíes sintieron verdadera afinidad, lo que habría facilitado la interacción. Ambos podrían haber competido por erigirse como curadores, obradores de milagros y santos. Incluso es posible que hayan frecuentado los mismos sitios y hasta hayan llegado a ejecutar prácticas similares en materia de control respiratorio. Diversas fuentes atestiguan que el intercambio entre musulmanes y yoguis fue duradero y contó con bastante actividad durante el periodo mogol. 'Abd Al-Quddus Gangohi, un sheik chishtiya en India en tiempos de Babur (s. xvi), sabía sobre hathayoga y gustaba de la poesía en hindi, en especial del tipo nirguñi que invocara a Gorakh o a Nirañjan (Gold, 1987). Incluso Gangohi a veces usaba el psuedónimo de "Alakh-das" (Aquil, 2007). A partir del siglo XviI comenzaron a circular fuera de India traducciones al persa, el árabe y el turco otomano del Amṛtakuṇụ, un texto sobre el control de la respiración debido al interés que los sufíes tenían por el tema. El mismo príncipe Dara Shikoh (s. XviI) escribió sobre ello. Pero ya desde el siglo XIv los autores musulmanes habían prestado atención a dicha práctica. Muhammad ibnMahmudAmuli, médico iraní, incluyó en su tratado enciclopédico una sección 
sobre el control respiratorio, sobre los tipos de aliento y sobre el sistema de chakras (Ernst, 2012).

Ahora bien, resulta importante mencionar que la interacción entre sufíes y yoguis figura sobre todo en fuentes sufíes, en particular la hagiografía. Un caso típico es la competencia para demostrar las respectivas facultades sobrenaturales. Dos ejemplos son el del yogui Ajaypal Nāth, quien junto con varios discípulos hace frente a Muin al-DinChishthi, recién llegado de Meca y que trataba de convertir al islam al dios de un templo (Ernst, 2005), y en un texto nizari del siglo xviII, donde Guru Hasan Kabiruddin demuestra su superioridad sobre Kanhapa (Khan, 2000). El mismo motivo aparece en las leyendas sobre los poetas santos Kabir, Anantadas o Nānak, que por lo general compiten contra Gorakh. Desde luego, parte del asunto radica en verificar qué términos emplean estos autores y textos para referirse a los yoguis. Asimismo, se debe revisar de qué manera lo hace el Dabestan-e Mazaheb, o Escuela de las religiones, el famoso texto indio escrito en lengua persa a mediados del siglo xviI. Claramente el vocablo más recurrido es jogī, la variante vernácula del norte de la India de la palabra sánscrita. Entre las excepciones de la hagiografía nāth, cabe destacar a Ratannāth, quien, según las fuentes, fue un discípulo de procedencia musulmana y maestro de GogāPīr, un personaje importante en el folclor de Rajasthan. Concretamente se sabe que las sectas HandiPharang y JafirPīrs constituyen ramas musulmanas de naths (Ernst, 2005; Briggs, 2001).

\section{La construcción de una identidad}

Si el término nāth antes del siglo XviII no se refiere a una corporación ascética, ¿cómo leer su incidencia en textos anteriores? Los contextos pueden variar, ciertamente, y en muchos casos no querrá decir más que "señor", usado como una especie de título honorífico o incluso como apellido. Pero cuando la palabra aparece en textos asociados con los yoguis, podría no ser el caso. Uno de los textos tempranos sobre hathayoga, y que en algún momento se atribuyó a Gorakh, es el Yogabija. Allí encontramos las siguientes palabras:

Este ha de ser el camino [que] concede las siddhis y escinde la red de Māyā; que aniquila el nacimiento, la vejez, la muerte y la enfermedad; y que otorga la felicidad y merced al cual los que están atados se liberan; [éste es] el supremo camino de la realización. Te hablaré del näthamārga por el que preguntas, Oh Sureśvarī, pues te aprecio $(6,7)$.

2. siddhamārgenalabhyetanānyathāśivabhāṣitam (Yogabïja, 8)

3. ādināthamahāmārgaekaeva hi nānyathā (Yogabīja, 154).

$84<$ Universidad de San Buenaventura, Cali - Colombia
Aquí hay que reflexionar con cuidado: "Nātha-mārga", ¿quiere decir el "camino del Señor"? Si Ādinātha (Siva) figura en las lista de Nāthas proverbiales, ¿cómo interpretar un apelativo en relación con el otro? Shiva es, literalmente, el "primer nātha, o seńor". Por otro lado, es Shiva quien está hablando aquí a su esposa; sería algo raro (aunque no imposible) que Shiva dijera te mostraré el camino de Shiva, en vez de decir el camino que lleva a mí o el camino que yo he enseñado.Un texto como el Yogabija no dice transmitir el camino de Siva-como-primer-nātha, sino el "camino nātha", o "de los nāthas".

En otra parte, la práctica también es llamada el "camino de los siddhas pronunciado por Shiva", una expresión que funciona como sinónimo de nāthamārga. ${ }^{2}$ Desde las tradiciones puránicas, tántricas y budistas, siddha se refiere a un perfecto practicante de métodos usualmente relacionados con alguna forma de yoga, pero también de magia. Hacia el final del texto, el dios dice que: "este gran secreto, el Yogabīja, el cual he esclarecido, debe ser difundido entre los devotos de Śiva que siguen el nāthamārga"(Yogabīja, 189).

La lectura más natural parece sugerir que la enseñanza contenida en este texto debe ser impartida a aquellos śaivas que siguen el camino de los perfectos yoguis (nāthas). Esta lectura es distinta de la que encontramos no muy lejos del final del Yogabija, donde explícitamente se menciona el àdināthamārga, es decir el camino del Primer nātha, o Siva. ${ }^{3}$ Tal vez nātha se empezó a utilizar como sinónimo de siddha y gradualmente se convirtió en título exclusivo de estos yoguis. La conclusión lógica a partir de estas citas es que el camino de los siddhas/nāthas deriva del dios y, por lo tanto, ni siddha ni nätha equivalen a Shiva.

Uno de los tratados en materia de yoga más difundidos es el Hathapradīpikā, un texto del siglo xv. Este cita unos cinco versos del Yogabija, que a su vez recoge unas nueve estrofas del Gorakșaśataka, cuya fecha probable de composición es 1400 e.c. (Mallinson, 2012). Así que el Yogabija posiblemente sea del 1450, lo cual hace pensar que para mediados del siglo $\mathrm{xv}$, al menos en algunas tradiciones yoguis, ya existía una forma cuando menos incipiente de denominar a los adeptos de hațhayoga y de Gorakh como nāthas. Además, resulta interesante advertir que si el Gorakșaśataka y el Yogabīja corresponden al periodo de 1400-1450, se podrían haber escrito más o menos durante la incursión de Timur en el norte de India y durante la dinastía sayyida. 
El argumento de Mallinson (2011, passim) es que antes de los siglos XVII-XVIII, la palabra nātha se refería invariablemente a la realidad absoluta y no a un militante de las varias escuelas de hathayoga. De regreso al caso de QuddusGangohi, al discutir la teoría de wahdat-ulwujuden en su Rushdnama cita varios versos en hindi medio donde invoca a Gorakh y a Nātha. De las seis veces que aparece $n \bar{a} t h a$, sugieren algunos especialistas, parece que al menos una vez denota al perfecto siddha (Aquil, 2007), justo lo que parece suceder en Yogabija. Si QuddusGangohi escribió el Rushdnama en su juventud, el texto correspondería a la segunda mitad del siglo xv, tal vez apenas algunas cuantas décadas después del Yogabija y quizá contemporáneo del Hațhapradīpikā.

\section{La tradición hagiográfica y literaria}

Otro modo de "medir" el uso de nātha como sinónimo de yogui de la escuela de Gorakh es rastrear las apariciones o menciones de listas de los personajes santos para dicha tradición, a saber los nueve nāthas. Es bastante probable que la lista no haya sido fijada sino hasta un momento más bien tardío, pero sin duda figura desde hace varios siglos en distintas fuentes. El Gurü granth sähib (ca. XVII) -las escrituras de los sikhs- posee referencias a los nueve nāthas, lo que debe implicar que la noción de un grupo de nueve antecede a la consolidación del SikhPanth. En el mismo siglo, en un poema devocional en memoria de Kabir, el poeta Malukadas menciona a los nueve nāthas junto con los ochenta y cuatro siddhas (Lorenzen, 1996).

Tanto näthas como siddhas, en un contexto como este, casi por necesidad son apelativos que se refieren a los practicantes de hathayoga relacionados con Matsyendra, Gorakh, Kanhapa y Caurangi, entre otros. Kabir en el siglo $\mathrm{Xv}$, antes que los autores sikhs y malukadas, también refiere por nombre a Gorakh y otros jogis. Puesto que los yoguis también compusieron poesía religiosa, es primordial buscar allí. La colección más afamada se conoce como Gorakhbāni, aunque posiblemente no todos los versos sean realmente obra de Gorakh. Puesto que en estos poemas no hay mención por nombre a ninguno de los poetas nirgunī, parece seguro afirmar que al menos la mayoría de estos versos anteceden a los nirgunī sants.

En el pad 38 del Gorakhbänī (Barthwal, 1994), Gorakh -o quien haya compuesto el verso- menciona a los nueve näths y los ochenta y cuatro siddhas. Estos personajes, a diferencia de los budistas, jainas, musulmanes, vaișnavas y otros religiosos, se distinguen por la práctica del hațhayoga, mientras que los otros encienden lámparas, hacen ofrendas de frutos y flores, se dejan crecer el cabello, se untan de cenizas o recitan libros sagrados. La citada lista de ochenta y cuatro siddhas tal vez cobró autoridad sobre todo a partir del recuento hagiográfico de Abhayadatta en el siglo XIII; es decir, antes de la composición del Hațapradīikikä, el Yogabija o el Gorakșaśataka. Significativamente, en esa lista de reputados siddhas, o maestros yoguis del budismo vajrayāna, se mencionan a Gorakh, Mina (Matsyendra), Kanhapa, Caurangi y Jalandhar, figuras prominentes de la hagiografía nāth. El poema aludido parece ser posterior a la obra tibetana; quizá contemporáneo, pero difícilmente anterior.

A expensas de que se lleve a cabo un trabajo filológico mucho más preciso y detallado, se puede afirmar que estos poemas anteceden la obra de Kabir (ca. siglo xv). Es decir, que los Gorakbbānī pueden datar del siglo xIv. Lo que resulta significativo de este y otros versos es, en primer lugar, el hecho de que para el autor la categoría de yoguis es diferenciable de hindúes y de musulmanes -un asunto que también elabora Kabir (cf. Lorenzen, 2011); y en segundo lugar, que ya existe una noción arraigada de un grupo de nueve näthas asociado con los practicantes de hathayoga. El verso citado explícitamente menciona que estos nāths y siddhas recurren a la práctica de āsanas, una de las marcas distintivas del hatha desde sus inicios. Parece más bien difícil entender que en este verso nau nātha signifique simplemente "nueve señores o amos", entendido como preceptor espiritual en general. Cabe considerar que el término está cargado de una idea que quizá se pueda parafrasear como "jefe del (hațha) yoga".

\section{Deducciones preliminares}

Las preocupaciones aquí expresadas sugieren varias inquietudes. Por un lado, resulta evidente la importancia de hacer estudios más profundos de las dinámicas sociorreligiosas e historiográficas durante el sultanato de Delhi, para acreditar mejor la importancia, la alternancia o la competencia entre distintos grupos religiosos y evaluar sus relaciones con los gobernantes en turno. En particular, vale la pena determinar si alguna de las dinastías permitió con mayor facilidad el desarrollo de grupos religiosos no islámicos, ya sea por debilitamiento político u otras razones. Por otro lado, también es necesario rastrear en las posibles regiones donde pudo haber surgido Gorakh (Punjab, el Decán, Bengala o Nepal; cf. Mallinson, 2011) para hallar documentos que ofrezcan evidencias más fiables acerca de su contexto y, en consecuencia, afianzar el primer eslabón de la historiografía nāth. En el caso del Decán, por ejemplo, podría ser significativo que la denominación religiosa 
más común era la chiita, en contraste con el sultanato de Delhi que fue sunita en su mayoría.

Por último, también surge una incógnita: ¿̨los sufíes hallaron afinidades y se retroalimentaron de los yoguis, o más bien fue la expansión de centros sufíes lo que, al menos en parte, favoreció la conformación de la orden de los nāths? En otras palabras, bien valdría la pena sondear la posibilidad de que fue justamente el contacto con algunas órdenes sufíes lo que aportó a los yoguis las herramientas argumentativas u organizativas para, por un lado, deslindarse cada vez más del ámbito tántrico -una inquietud recurrente en la literatura hagiográfica- $y$, por el otro, idear modos de construirse una identidad más o menos autónoma. $\mathrm{Al}$ respecto, resulta significativo que más de un líder nāth haya recibido el título de pìr, y que varios personajes mogoles hayan sostenido entrevistas con yoguis casi en la misma medida que con líderes de órdenes sufíes. Justamente el mostrarse -cuando menos en teoría- como un grupo con una identidad distinta del resto de los hindúes pudo haber favorecido el intercambio con los ascetas musulmanes, el movimiento más o menos libre entre la población en general y la conservación de un hálito especial de santidad que tal vez no siempre pudieron preservar los pandits frente a los sultanes y los posteriores monarcas mogoles. En un sentido, esa misma naturaleza amorfa, abierta y universalista caracterizó el éxito de los poetas nirgunis. De todos estos movimientos poético-religiosos, claro está, el único que logró explotar verdaderamente la diferenciación identitaria fue Nanak, quien terminó por fundar una religión independiente y no solo una secta o grupo absorbido por el hinduismo.

Vale la pena recordar que Kabir ca. 1440-1518 (entre las dinastías Sayyid y Lodi) y Surdas ca. 1474-1584 (entre la dinastía Lodi y el reino de Akbar) florecieron bajo el sultanato de Delhi, de manera simultánea con órdenes de sufíes. Significativamente, por otra parte, (Prasad, 1990) parece que durante el sultanato de Delhi, entre los títulos para referirse al sultán en las inscripciones sánscritas se encontraba la palabra nāth. Al mismo tiempo, tampoco se puede descartar la posibilidad de que no hayan sido solo los yoguis quienes comenzaron a utilizar el término nātha para referirse a ellos mismos; bien podría haber sido la población en general, o los sufíes o los gobernantes musulmanes. Al respecto, podría ser relevante que el padre Antonio Monserrate, jesuita en la corte de Akbar, durante una visita real a la cueva de Gorakh Tilla recalcara que todos los yoguis allí residentes se llamaban nāth.

Tras criticar severamente a Balnāth, cabeza de la congregación de yoguis, Monserrate escribe: "Este perverso ha engatusado a los tontos hindúes para que lo veneren y le llamen con varios nombres que terminan en "nat" [nātha], tales como Manquinat, Septenat, Jagarnat, etc." (Pinch, 2012). Sería sensato suponer que la insistencia en usar el apelativo de näth hubiera podido desembocar en que dicho apelativo terminara por convertirse en una marca identitaria. En todo caso, este punto requiere una investigación más minuciosa en las fuentes originales, no solo los registros de los misioneros y los textos de los nāths, sino en documentos oficiales, bitácoras de viaje y la literatura de movimientos religiosos coetáneos entre los siglos XIII y XVII.

Aun si aceptamos que la orden de yoguis, autodefinida como Nāth Panth o Nāth Sampradaya, no es anterior al siglo XVIII aproximadamente. Ello no necesariamente cancela el hecho de que los yoguis anteriores no se concibieran a sí mismos como nāths, quizá al principio solo en el sentido genérico de "señores del yoga". Cabe pensar que la alta recurrencia del título näth anexado al nombre de un sinnúmero de yoguis (legendarios e históricos) sugiere que la conciencia del asceta hathayóguico ya perfilaba una distinción crucial y cualitativa con respecto de otros ascetas y yoguis. No solo eran yoguis genéricos; eran yoguis de tipo nāth. Un yogui seguidor del camino de Patañjali, por ejemplo, no es un $n \bar{a} t h$ ni se sabe que exista alguna descripción como tal en alguna fuente. En parte, la generación de una lista de nueve näthas proverbiales parece reforzar la incipiente conciencia nāth antes de la era moderna. La orden podría haberse "unificado" o "institucionalizado" hacia el siglo XviII, pero quizá solo porque ya se había desarrollado una conciencia más o menos clara de que estos yoguis eran distintos de otros yoguis o renunciantes y el apelativo que más los diferenciaba era nāth.

\section{Referencias}

Aquil, R. (2007). Sufism, Culture and Politics. Afghans and Islam in Medieval North India. Oxford: Oxford University Press.

Barthwal, P. (1994). GorakhBāṇī. Hindī sāhitya sammelan: Allahabad.

Bhattacharyya, N. N. (1996). Ancient Indian Rituals and their Social contents. Delhi: Manohar.

Briggs, G. W. (2001). Gorakhnāth and the Känphața Yogiss. Motilal Banarsidas: Delhi/Varanasi/Patna.

Bouy, C. (1994). Les Nātha-yogin et les Upanișads. Étude d'histoire de la littérature hindoue. Paris: Editions de Boccard.

Champion, C. (1995). Entre la caste et la secte: un kissa du repertoire des BharthariJogi musulmans de la región de Gorakhpur. In M. L. Reiniche \& H. Stern (Eds.), Le ruse du

$86<$ Universidad de San Buenaventura, Cali - Colombia 
salut. Religion et politiques dans le monde indien, Purușārtha 17 (pp.25-42). Paris: Editions de l'Ecole des hautes Etudes en Sciences Sociales.

Dvivedi, H. (1996). Nāth-Sampradāy. Lok Bhāratī Prakāśan: Ilāhābād.

Dvivedi, H. (2004). Kabir's Place in Indian Religious Practice. In D. Lorenzen (Ed.), Religious Movements in South Asia, 600-1800 (pp.269-87). Oxford/NY/Delhi: Oxford University Press.

Ernst, C. (2005). Situating Sufism and Yoga. Journal of the Royal Asiatic Society, 15(1), 15-43.

Ernst, C. (2007). Accounts of yogis in Arabic and Persian historical and travel texts. Jerusalem Studies in Arabic and Islam, 33, 409-26.

Ernst, C. (2012). A Fourteenth-Century Persian Account of Breath Control and Meditation. En D. G. White (Ed.), Yoga in Practice (pp.133-139). Princeton: Princeton University Press.

Gold, D. (1987). The Lord as Guru. Hindi Sants in North Indian Tradition. NY/Oxford: OUP.

Gold, D. (1992). Ascenso y caída del poder de los yoguis: Jodhpur 1803-1842. Estudios de Asia y África, 27(1), 9-27.

Gold, D. (1995).The Instability of the King: Magical Insanity and the Yogi's Power in the Polictis of Jodhpur. En D. Lorenzen (Ed.), Bhakti Religion in North India (pp.120-132). Albany, NY: State University of New York Press.

Goswamy, B. N., \& Grewal, J. S. (1967). The Mughals and the Jogis of Jakhbar. Simla: Indian Institute of Advanced Study.

Khan, D. (2000). Conversation Between Guru Hasan Kabiruddin and Yogi Kahipa: Tantra revisited by isma' ili Preachers. In
D. G. White (Ed.), Tantra in Practice (pp.285-95) Princeton: Princeton University Press.

Lorenzen, D. N. (1996). Praises to a Formless God. NY, Albany: SUNY.

Lorenzen, D. N. (2011). Religious Identity in Gorakh and Kabir: Hindus, Muslims, Yogis, and Saints. In D. Lorenzen \& A. Muñoz (Eds.), YogiHeroes and Poets. (pp.19-49). Albany, NY: State University of New York Press.

Mallinson, J. (2011). Nāth Sampradāya. Brill Encyclopedia of Religion. Leiden: Koninklijke Brill.

Mallinson, J. (2012). The Original Gorakșaśataka. En D.G. White (Ed.), Yoga in Practice (pp.257-272). Princeton: Princeton University Press,

Muñoz, A. (2011). Yoguis y sufíes: diálogos indoislámicos, Humania del Sur, 6(10) 97-111.

Pinch, W. (2012). Nath Yogis, Akbar, and Balnath Tilla. In D. G. White (Ed.), Yoga in Practice (pp.273-88). NJ: Princeton University Press.

Prasad, P. (1990). Sanskrit Inscriptions of Delhi Sultanate, 11911526. Delhi: Oxford University Press.

Sarkar, J. N. (1985). A Study of Sufism. Its background and syncretic significance in Medieval India. Indo-Iranica, $38(1-2), 1-24$.

White, D. (2009). Sinister Yogis. IL: The University of Chicago Press.

Yogabīja. (1982; V.S. 2039). Ed. RāmlālŚrīvāstav. Gorakhpur: Gorakhnāthmandir.

YogaBija. (1985; V.S. 2042). Edited and translated by M.M. Dr. BrahmamitraAwasthi. Delhi: Swami Keshananda Yoga Institute. 